\title{
Large Eddy Simulation of the Wind Field and Pollution Dispersion in Building Array
}

\author{
R. F. Shi ${ }^{1}$, G. X. Cui ${ }^{1 *}$, C. X. Xu' ${ }^{1}$, Z. S. Zhang ${ }^{1}$, Z. S. Wang ${ }^{2}$ \\ ${ }^{1}$ Department of Engineering Mechanics, Tsinghua University, Beijing 100084, China \\ ${ }^{2}$ Department of Civil and Environment Engineering, University of Macau, Macao, China
}

\begin{abstract}
The air quality is one of the significant issues in sustainable development of modern city. In the paperthe Large Eddy Simulation (LES) is used to predict the wind field and pollution dispersion in residence district. The flow in residence district is characterized by the complicated geometry, vortex patterns, unsteadiness etc.; hence the LES is the suitable method for the flow. The finite volume method with fourth order accuracy is utilized for numerical simulation and immerse boundary method (IBM) is applied to the solid boundary condition. The numerical scheme has been validated for a number of complex flows ( $\mathrm{Xu}$ et al. 2006). A number of testing cases have been performed and the results are compared with experimental data in good agreement. One of the testing cases is an array of cubic buildings with height $H$, length $B$ and width $W$ of $0.12 \mathrm{~m}$. The flow Reynolds number based on the free stream velocity and the height of the building is equal to $4 \times 10^{6}$. A point concentration source is located at $4 \mathrm{~W}$ in front of the building array. The dynamic Smagorinsky model is used for the subgrid stress. The predicted results are compared with the wind tunnel measurements by Davidson et al. (1996). Figure 1 and 2 shows the typical wind speed distributions and the mean concentration at $H / 2$ and $L_{a y} / 2$, They are in good agreement with experimental data. The results indicate that the proposed numerical scheme of LES is capable of predicting wind field and pollution dispersion in residence district. Moreover numerical simulation can provide more information about the flow pattern and concentration distribution for better understanding the phenomena.
\end{abstract}
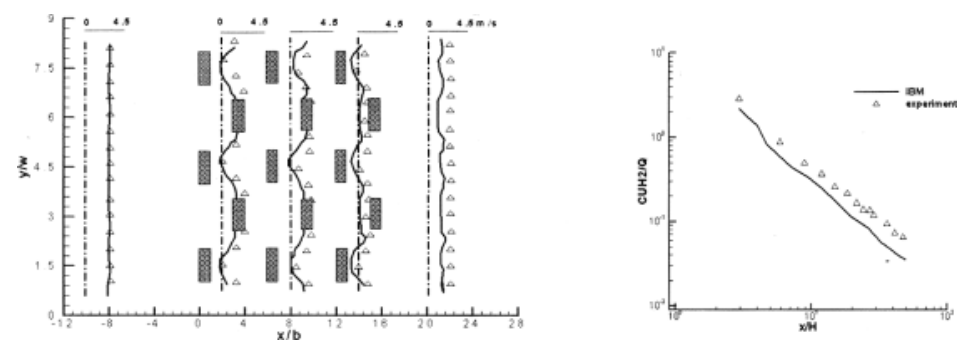

Figure 1 Lateral distribution of Streamlines velocity Figure 2 Concentration distribution

\section{REFERENCES}

1. Davidson M.J, et al. Wind tunnel simulation of plume dispersion through groups of obstacles. Atmospheric Environment, 1996;30(22):3715-3731

2. Xu L, et al. High accurate finite volume method for large eddy simulation of complex turbulent flows. International Journal of Turbo \& Jet-Engine, 2006;23(3):191-210 